\title{
Self-distribution-function procedure in elastic incoherent neutron scattering for biosystems molecular motion characterization
}

\author{
Salvatore Magazù ${ }^{\mathrm{a}, *}$, Federica Migliardo ${ }^{\mathrm{a}, \mathrm{b}}$, Antonio Benedetto ${ }^{\mathrm{a}}$, Miguel Gonzalez ${ }^{\mathrm{c}}$ and \\ Claudia Mondelli ${ }^{\mathrm{c}}$ \\ ${ }^{a}$ Dipartimento di Fisica, Università di Messina, Messina, Italy \\ ${ }^{\mathrm{b}}$ Laboratoire de Dynamique et Structure des Matériaux Moléculaires, UNESCO-L'Oreal University \\ of Lille I, Villeneuve d'Ascq, France \\ ${ }^{\mathrm{c}}$ Consiglio Nazionale delle Ricerche - Istituto Officina dei Materiali, Institut Laue-Langevin, \\ Grenoble, France
}

\begin{abstract}
In the present contribution we present a new procedure for the Mean Square Displacement (MSD) determination from Elastic Incoherent Neutron Scattering (EINS) where the connection between the Self-Distribution Function (SDF) and the measured EINS intensity profile is highlighted. We show how the SDF procedure allows both the total and the partial MSD evaluation, through the total and the partial SDFs. The procedure is applied on EINS data collected, by the IN13 backscattering spectrometer (ILL, Grenoble), on aqueous mixtures of sucrose and trehalose.
\end{abstract}

Keywords: Molecular motions, self-distribution function, mean square displacement, resolution effects, elastic incoherent neutron scattering, homologous disaccharides

\section{Introduction}

It is well known that the characterization of the different molecular processes involved in the dynamics of some molecular and macromolecular systems of biophysical interest, such as for example bioprotectant/water mixtures, pure and hydrated polymeric systems, hydrated and crystalline proteins, can be effectively investigated by evaluating the Mean Square Displacement (MSD) [1] from Elastic Incoherent Neutron Scattering (EINS) data collected by varying temperature, energy resolution, wavevector and energy range and by using isotopic labelling. More specifically, a wide temperature range can facilitate the spectral separation of different molecular processes according to their time-scale, while the temperature dependence of the measured elastic intensity can provide information about the involved activation energies and, thus, on the local potentials. On the other hand, a wide $Q$-range, such as that of the IN13 backscattering spectrometer at the Institute Laue-Langevin (ILL) in Grenoble with a $Q$-range extending up to $5 \AA^{-1}$, can allow to achieve a molecular assignment based on spatial features.

\footnotetext{
*Corresponding author: Salvatore Magazù, Dipartimento di Fisica, Università di Messina, C. da Papardo n 31 , P.O. Box 55, Vill. S. Agata, 98166 Messina, Italy. Tel.: +39 0906765025; Fax: +39 090395004; E-mail: smagazu@ unime.it.
} 
In the framework of the Gaussian approach, the MSD can be obtained by a linear regression in the so-called Guinier plot (where the logarithm of the elastic intensity is plotted as a function of $Q^{2}$ ) for a set of points close to $Q=0$. However this approach does not allow to separate the different contributions related to a specific spatial domain and furnishes MSD values which are dependent on the $Q$-range used for the MSD evaluation. The aim of the present contribution is to improve the formulation of the Self-Distribution Function (SDF) procedure, proposed in a previous works [3-9] for evaluating the total and the partial MSDs. On that score we will apply the procedure to EINS data collected by using the IN13 spectrometer at ILL on aqueous mixtures of two homologous disaccharides (i.e. sucrose and trehalose).

It is well known that the scattering law $S(Q, w)$ is connected, in Planck's units, through its time Fourier transform $\left(F_{t}\right)$, to the intermediate scattering function $I(Q, t)$, and, through its space-time Fourier transform $\left(F_{r, t}\right)$, to the time-dependent spatial correlation functions $G(r, t)[2,11]$. The scattering law $S(Q, w)$ is proportional to the observed neutron intensity, the proportionality factors being represented by the incident and outgoing neutron wave vectors, the number of scattered atoms and the scattering cross-sections (e.g., $\sigma_{\text {inc }}($ hydrogen $)=81.0$ barn and $\sigma_{\text {inc }}($ deuterium $)=2.2$ barn $)$. For samples with mainly incoherent scattering cross-sections, the relevant correlation function is the $\operatorname{SDF} G_{s}(r, t)$ which, following Van Hove [10], represents the probability to find the same particle at distance $r$ after a time $t$.

In this framework, due to the energy instrumental resolution $\Delta w$, the experimentally accessible quantity is the scattering function $S_{R}(Q, w, \Delta w)$, i.e. the convolution of the scattering law with the instrumental resolution function $R(w, \Delta w)[2,11]$.

\section{Experimental section}

Experimental data were collected by the IN13 spectrometer at ILL which is characterized by a relatively high energy of the incident neutrons $(16 \mathrm{meV})$. The experimental set up was: incident wavelength $2.23 \AA$; $Q$-range $0.28-4.27 \AA^{-1}$; elastic energy resolution (FWHM) $8 \mu \mathrm{eV}$. Raw data were normalized to unity at $Q=0.28 \AA^{-1}$. Measurements were performed in the temperature range of $20-300 \mathrm{~K}$ on hydrogenated trehalose and sucrose in $\mathrm{H}_{2} \mathrm{O}$, purchased by Sigma-Aldrich, at a weight fraction value corresponding to 19 water molecules for each disaccharide molecule.

\section{Self-distribution function procedure}

Following previous works [3-9] the normalized space Fourier transform of measured EINS intensity profile corresponds to the SDF evaluated at an equivalent time $t^{*}, G^{\text {self }}\left(r, t^{*}\right)$, which is connected to the instrument resolution energy and to the system characteristic time. In the framework of the SDF procedure, under the single process Gaussian ansatz, we obtain

$$
G^{\text {self }}\left(r, t^{*}\right) \propto F_{r}\left\{S_{R}(Q, \omega=0 ; \Delta \omega)\right\}=\sum_{n} A_{n} F_{r}\left\{\mathrm{e}^{-Q^{2} a_{n}}\right\} \propto \sum_{n} A_{n} G_{n}^{\text {self }}\left(r, t^{*}\right)
$$

in which $G_{n}^{\text {self }}\left(r, t^{*}\right)$ are the partial SDFs and $F_{r}$ is the spatial Fourier transform operator.

In this framework the $r^{2}$ mean value results (the $B_{n}$ terms are the normalized $A_{n}$ coefficients)

$$
\left\langle r^{2}\right\rangle=\int_{-\infty}^{\infty} r^{2} G^{\text {self }}\left(r, t^{*}\right) \mathrm{d} r=\sum_{n} B_{n} \int_{-\infty}^{\infty} r^{2} G_{n}^{\text {self }}\left(r, t^{*}\right) \mathrm{d} r=2 \sum_{n} B_{n} a_{n} .
$$


The SDF can be applied directly to the experimentally determined EINS profiles as well as to whichever function able to reproduce their behaviour; it represents an integral procedure which takes into account the global $Q$ behaviour and so doing it allows to reduce the error of the $Q \rightarrow 0$ extrapolation. In fact the MSD evaluation in the limit $Q \rightarrow 0$ yields [8]

$$
\begin{aligned}
\left\langle r^{2}\right\rangle & =-\left.2 \frac{\partial I(Q, t)}{\partial Q^{2}}\right|_{Q=0}=-\left.2 \frac{\partial}{\partial Q^{2}} \sum_{n} B_{n} \mathrm{e}^{-Q^{2} a_{n}}\right|_{Q=0}=\left.2 \sum_{n} B_{n} a_{n} \mathrm{e}^{-Q^{2} a_{n}}\right|_{Q=0} \\
& =2 \sum_{n} B_{n} a_{n} .
\end{aligned}
$$

In addition, the SDF procedure allows to separate the different MSD contributions when the partial SDFs are considered [3-9]

$$
\left\langle r^{2}\right\rangle_{n}=\int_{-\infty}^{\infty} r^{2} G_{n}^{\text {self }}\left(r, t^{*}\right) \mathrm{d} r=2 a_{n}
$$

Figure 1(a) and (b) show the obtained SDF and its partial contributions as a function of $r$ at $T=284 \mathrm{~K}$ for sucrose and trehalose, respectively. As it can be seen the different kinds of motion are spatially well separated within the accessible $Q$-range; furthermore the SDF very closely follows the first partial SDF in the range (0-0.5) $\AA$ and the second one in the range (0.5-5) $\AA$.

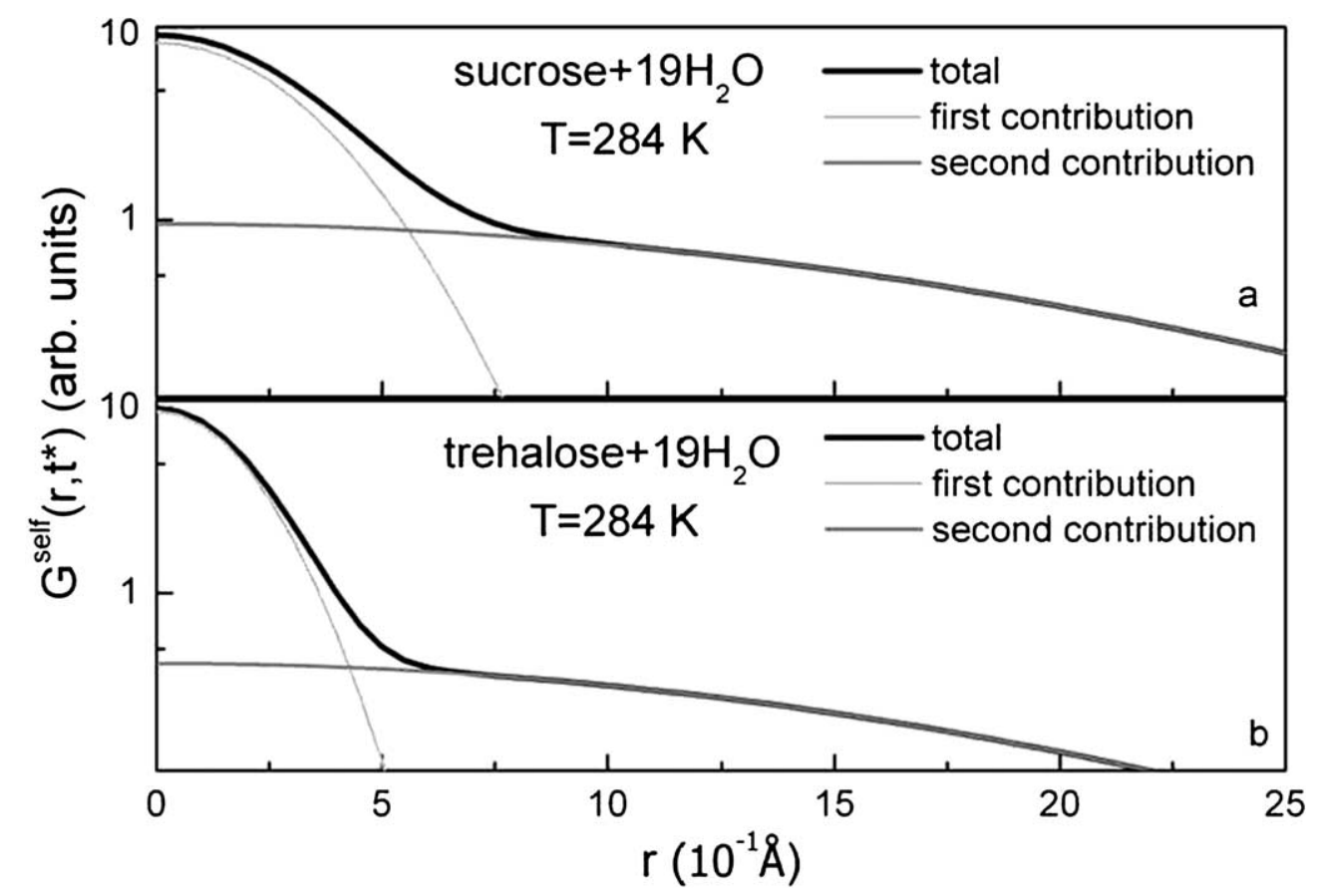

Fig. 1. SDF (black) together their different contributions (gray) for sucrose and trehalose at $T=284 \mathrm{~K}$. The different kinds of motion are spatially separated within the accessible $Q$-range and the total SDF closely follows the first partial SDF in the range $(0-0.5) \AA$ and the second one in the range $(0.5-5) \AA$. 


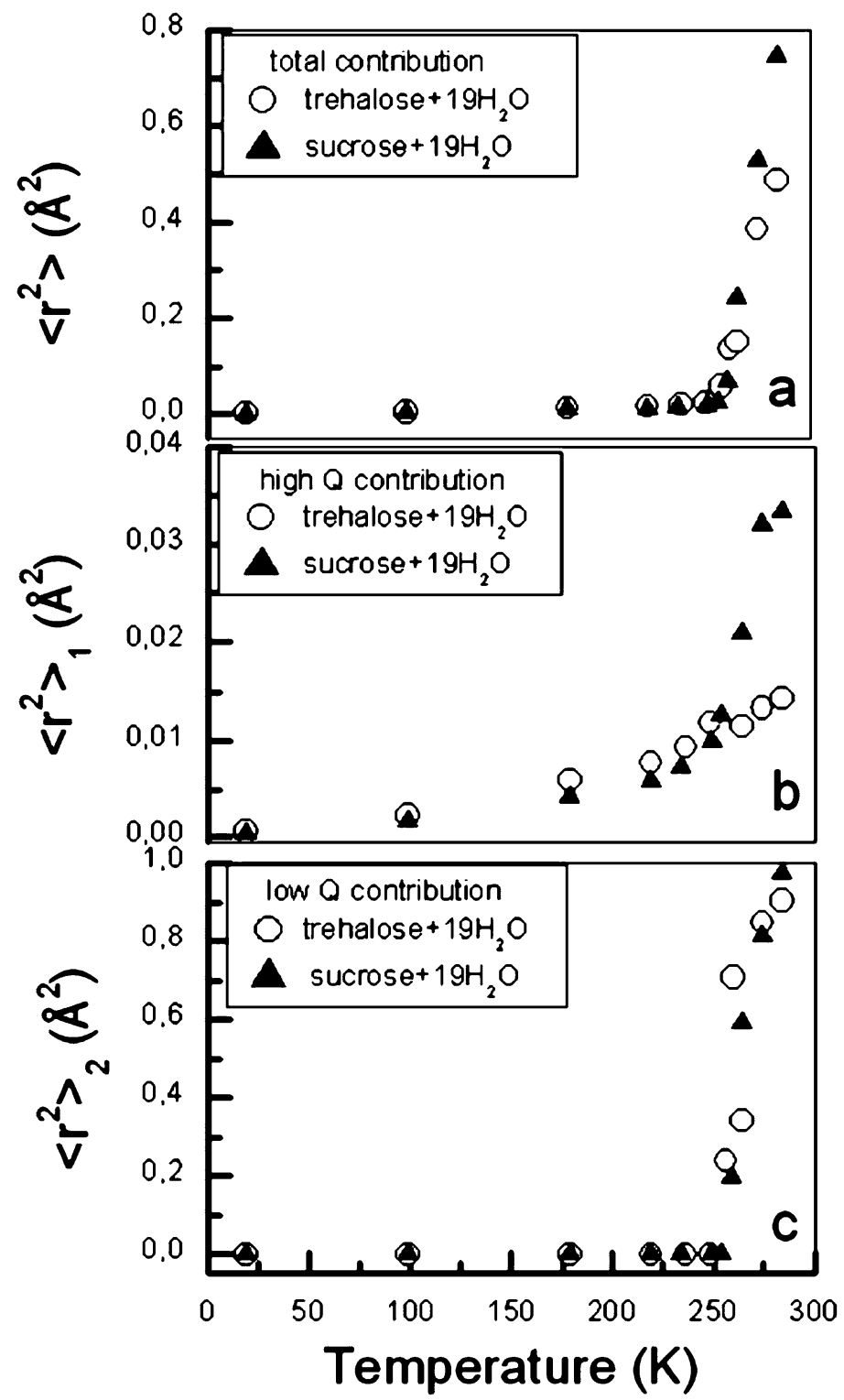

Fig. 2. MSD temperature behaviour for trehalose and sucrose in the temperature range 20-287 K. (a) The total contribution, (b) the partial high $Q$-contribution and (c) the partial low $Q$-contribution of MSD for sucrose and trehalose; as it can be seen, the partial MSD behaviours of sucrose and trehalose are equivalent in the high- $r$ domain, whereas they are different in the small- $r$ domain.

Figure 2(a) shows the total MSD, that taking into account Eqs (2) and (4), corresponds to a weighted sum of the partial MSD contributions [3-9]

$$
\left\langle r^{2}\right\rangle=\sum_{n} B_{n}\left\langle r^{2}\right\rangle_{n}
$$


Figure 2(b) and (c) show the partial contributions of the MSD for sucrose and trehalose, within the temperature range of 20-287 K, in the high- $r$ domain and in the small- $r$ domain, respectively. As it can be seen, the partial MSDs of sucrose and trehalose are equivalent in the high- $r$ domain, whereas they are differ in the small- $r$ domain. This circumstance suggests that the higher structure sensitivity of sucrose in respect to trehalose should be related to the smaller spatial observation windows. It is also important to observe that the dynamical transition temperature of the partial MSDs is equal for the two disaccharides and is equal to the dynamical transition temperature of the average MSD.

\section{Conclusions}

By applying the SDF procedure to water/homologous disaccharide mixtures, a different dynamical behaviour in the large $Q$-range region is pointed out at temperature values higher than about $250 \mathrm{~K}$. It emerges that the hydrogen bond imposed network of the water-trehalose mixture appears to be stronger in respect to that of the water-sucrose mixture and this result can justify the highest bioprotectant effectiveness of trehalose in comparison with sucrose.

\section{Acknowledgements}

The authors wish to acknowledge the Institut Laue-Langevin for dedicated beam time on IN13.

The present results have been obtained in the frame of a research project performed in collaboration with ESA, which financially supports this research.

\section{References}

[1] B. Alefeld, A. Kollmar and B.A. Dasannachrya, J. Chem. Phys. 63 (1976), 4415.

[2] M. Bee (ed.), Quasielastic Neutron Scattering, Adam Hilger, Bristol, 1988.

[3] A. Benedetto, S. Magazù, G. Maisano and F. Migliardo, International Conference on Complexity, Metastability and Nonextensivity, in: Complexity, Metastability and Nonextensivity, S. Abe, H. Herrmann, P. Quarati, A. Rapisarda and C. Tsallis, eds, Catania, Italy, 2007.

[4] S. Magazù, G. Maisano, F. Migliardo and A. Benedetto, Phys. Rev. E 77 (2008), 061802.

[5] S. Magazù, G. Maisano, F. Migliardo and A. Benedetto, J. Phys. Chem. 112 (2008), 8936-8942.

[6] S. Magazù, G. Maisano, F. Migliardo and A. Benedetto, J. Mol. Struct. 882 (2008), 1.

[7] S. Magazù, G. Maisano, F. Migliardo and A. Benedetto, Phys. Rev. E 79 (2009), 041915.

[8] S. Magazù, G. Maisano, F. Migliardo and A. Benedetto, Biochim. Biophys. Acta 1804 (2010), 49.

[9] S. Magazù, G. Maisano, F. Migliardo, G. Galli, A. Benedetto, D. Morineau, F. Affouard and M. Descamps, J. Chem. Phys. 129 (2008), 155103.

[10] L. Van Hove, Phys. Rev. 95 (1954), 249.

[11] F. Volino, Spectroscopic Methods for the Study of Local Dynamics in Polyatomic Fluids, J. Dupuy and A.J. Dianoux, eds, Plenum, New York, 1978. 


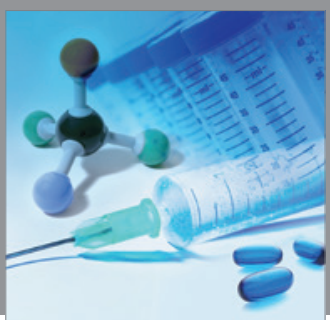

International Journal of

Medicinal Chemistry

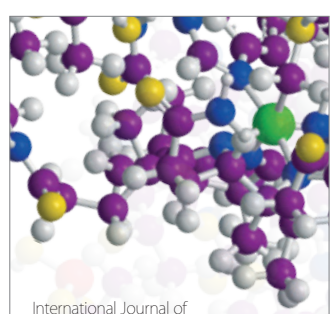

Carbohydrate Chemistry

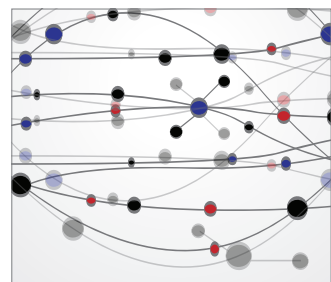

The Scientific World Journal
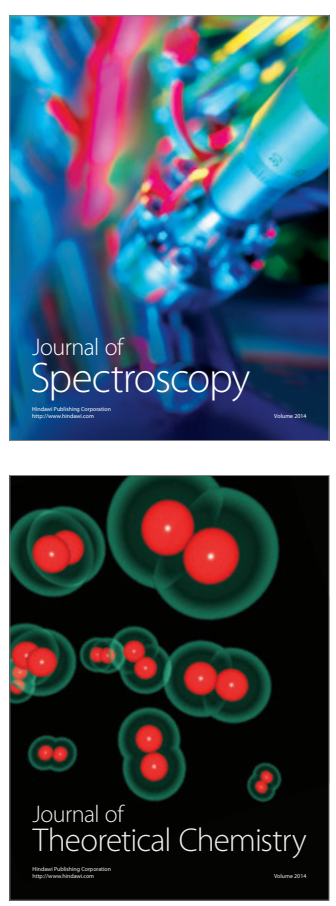
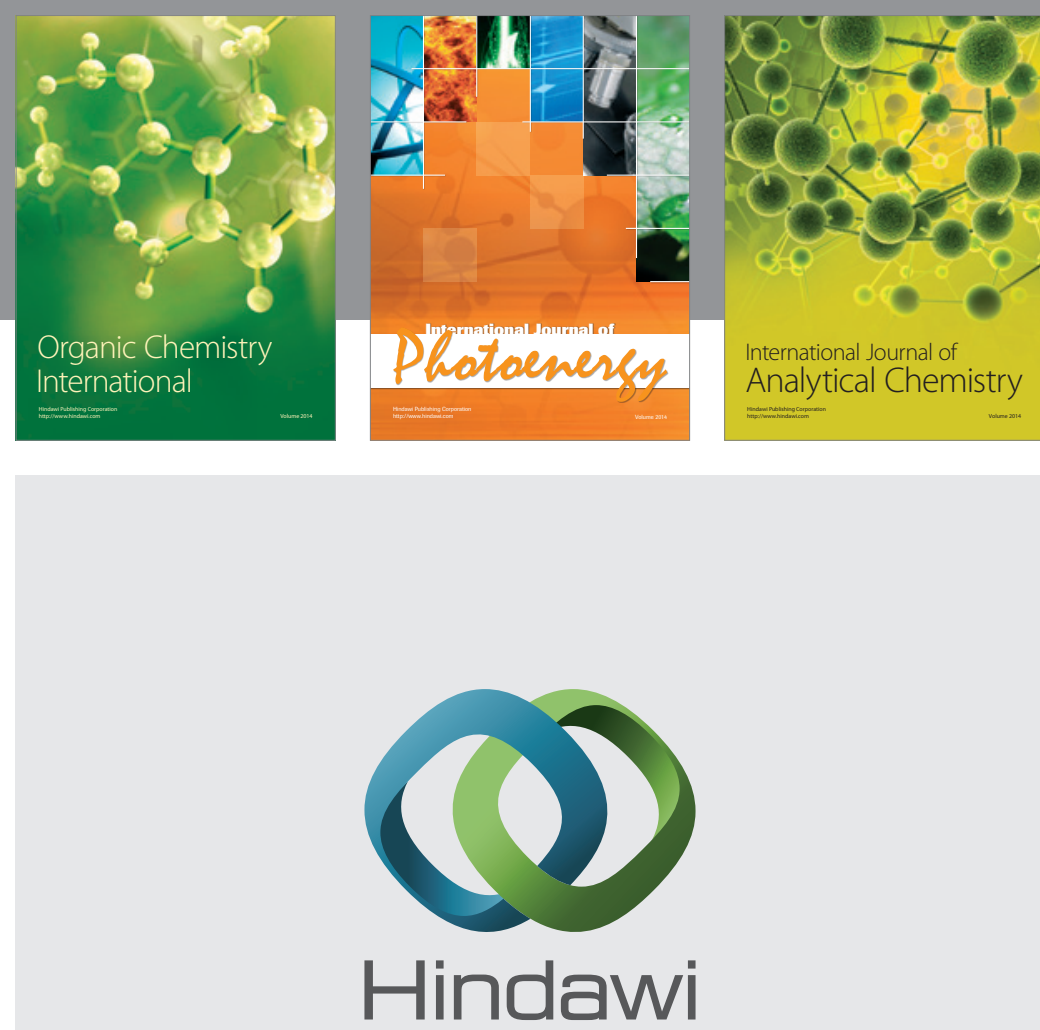

Submit your manuscripts at

http://www.hindawi.com
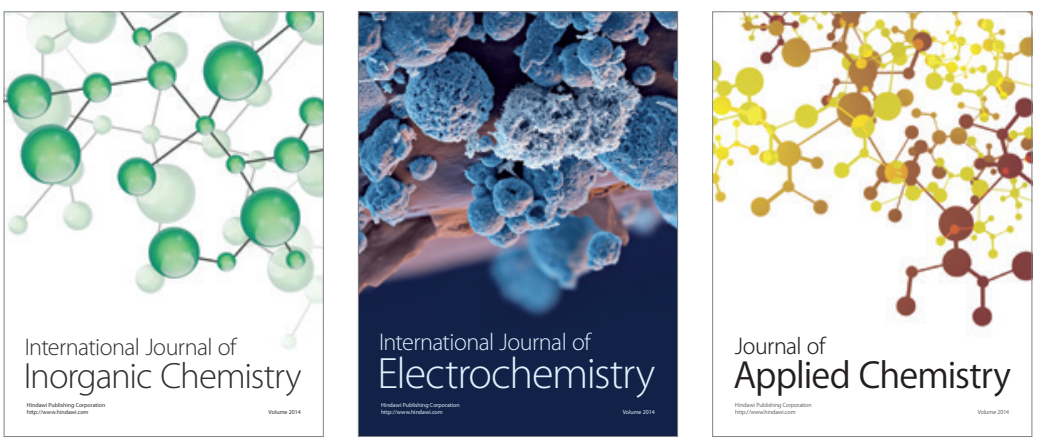

Journal of

Applied Chemistry
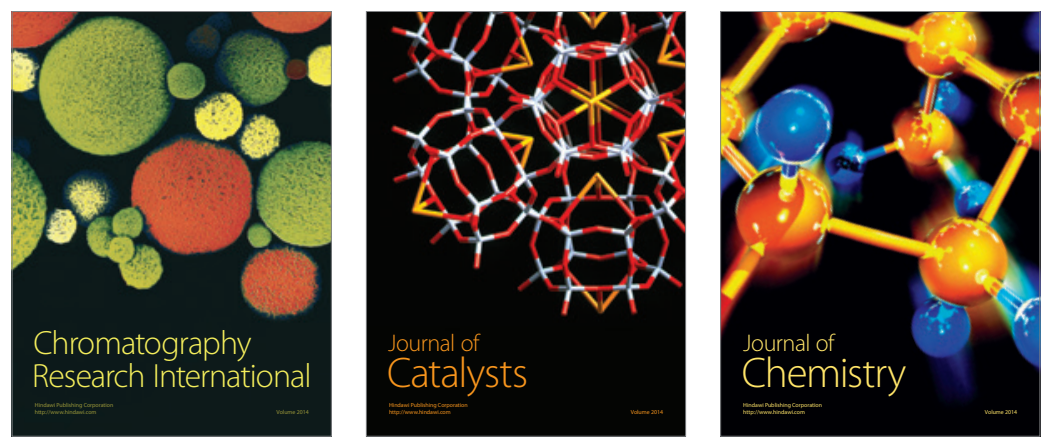
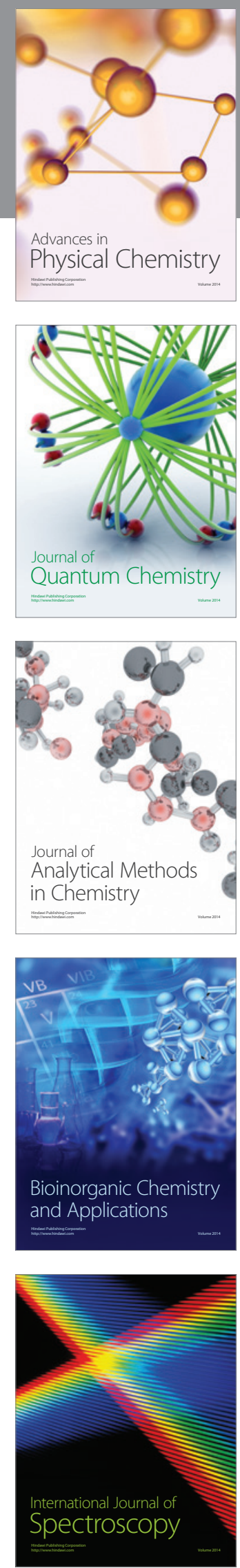